\title{
Applying the Coupled-Cluster Ansatz to Solids and Surfaces in the Thermodynamic Limit
}

\author{
Thomas Gruber, Ke Liao, and Theodoros Tsatsoulis \\ Max Planck Institute for Solid State Research, Heisenbergstrasse 1, 70569 Stuttgart, Germany \\ Felix Hummel \\ Institute for Theoretical Physics, Vienna University of Technology, \\ Wiedner Hauptstrasse 8-10, 1040 Vienna, Austria \\ Andreas Grüneis* \\ Institute for Theoretical Physics, Vienna University of Technology, \\ Wiedner Hauptstrasse 8-10, 1040 Vienna, Austria \\ and Max Planck Institute for Solid State Research, Heisenbergstrasse 1, 70569 Stuttgart, Germany
}

(Received 2 November 2017; revised manuscript received 19 February 2018; published 14 May 2018)

\begin{abstract}
Modern electronic structure theories can predict and simulate a wealth of phenomena in surface science and solid-state physics. In order to allow for a direct comparison with experiment, such $a b$ initio predictions have to be made in the thermodynamic limit, substantially increasing the computational cost of manyelectron wave-function theories. Here, we present a method that achieves thermodynamic limit results for solids and surfaces using the "gold standard" coupled cluster ansatz of quantum chemistry with unprecedented efficiency. We study the energy difference between carbon diamond and graphite crystals, adsorption energies of water on $h$-BN, as well as the cohesive energy of the Ne solid, demonstrating the increased efficiency and accuracy of coupled cluster theory for solids and surfaces.
\end{abstract}

DOI: 10.1103/PhysRevX.8.021043

Subject Areas: Chemical Physics,

Computational Physics,

Condensed Matter Physics

\section{INTRODUCTION}

Modern $a b$ initio methods to solve the electronic Schrödinger equation for real solids and molecules such as density functional theory or wave-function-based methods are becoming increasingly accurate and efficient [1-4]. However, in contrast to molecular systems, properties of solids and surfaces need to be calculated in the thermodynamic limit. The convergence towards the thermodynamic limit with respect to the number of particles is very slow, often exceeding the computational resources of even modern supercomputers. This is particularly the case for many-electron wave-function-based theories that allow for a systematic improvability upon the description of the electronic correlation energy. Nonetheless, these methods are becoming increasingly popular in theoretical physics as well as in chemistry to treat electronic correlation in

\footnotetext{
*andreas.grueneis@tuwien.ac.at
}

Published by the American Physical Society under the terms of the Creative Commons Attribution 4.0 International license. Further distribution of this work must maintain attribution to the author(s) and the published article's title, journal citation, and DOI. periodic condensed-matter systems with high accuracy [3-13].

Electronic correlation is, for the most part, a short-ranged phenomenon. The proper description of the wave-function shape at short interelectronic distances allows for capturing the largest fraction of the correlation energy in solids $[2,14]$. Significant progress has been achieved for manyelectron wave-function-based theories by exploiting the locality of electronic correlation in large molecules and solids. The development of so-called local correlation methods and embedding theories has improved their computational efficiency considerably [15-24]. However, theories that approximate long-range correlation effects such as van der Waals interactions must carefully be checked for convergence with respect to the employed cutoff parameters to allow for accurate and predictive $a b$ initio studies of real materials. This is of particular importance in condensed-matter systems where the accumulation of weak van der Waals interactions can become a non-negligible contribution to the property of interest, as, for example, in the case of the energy difference between carbon diamond and graphite or the adsorption of a water molecule on an $h$-BN sheet. Pairwise additive interatomic van der Waals interactions cause a $1 / N$ convergence of the electronic correlation energy per unit cell in insulating 
three-dimensional systems, where $N$ is the number of explicitly correlated atoms. However, in general, the exact form of these scaling laws depends on the dimensionality and electronic response properties of the system; e.g., the adsorption energy of molecules on two-dimensional insulating surfaces exhibits a $1 / N^{2}$ convergence. Moreover, we note that collective phenomena such as plasmons in metallic systems can also modify the observed scaling laws [25]. For these reasons, robust and reliable approximations to long-range correlation effects are nontrivial.

Many-body methods such as coupled cluster or configuration interaction theory can describe both long- and short-ranged electronic correlation effects with high accuracy. However, the scaling of the computational complexity of these theories with respect to system size is either of a high-order polynomial or an even exponential form. Therefore, it is difficult to treat long-range correlation effects in a computationally efficient manner using these theories. This has led to the development of various techniques that partition the correlation problem according to a predefined criterion such as the distance between electron pairs or fragment size. Local theories employ correlation energy expressions that depend on localized electron pairs, making it possible to treat long-distance pairs using computationally more efficient yet less accurate theories. Embedding theories typically aim at combining the computational efficiency of mean field theories for the long range, with the high accuracy of wave-function-based methods applied to small fragments only. In this work, we introduce an efficient method that seamlessly integrates long-range correlation effects for solids without any predefined criteria such as cutoff distance or fragment size. Our approach is inspired by structure factor interpolation techniques as performed in the field of quantum Monte Carlo theory [26]. However, in coupled cluster theory, the structure factor, being the functional derivative of the total energy with respect to the Coulomb kernel, is not directly available. Instead, we seek to interpolate the partial functional derivative of the coupled cluster correlation energy expression with respect to the Coulomb kernel. The interpolation scheme is chosen such that it is directly transferable to systems with arbitrary dimensions including solids and surfaces. Because of the adverse scaling of the computational complexity in coupled cluster theories, the proposed method allows for reducing the computational cost by several orders of magnitude without compromising accuracy compared to previous studies [4].

\section{THEORY}

The electronic correlation energy can be calculated in a plane-wave basis set using the following expression [27]:

$$
E_{c}=\left\langle\Psi_{0}\left|H-E_{0}\right| \Psi\right\rangle=\sum_{\mathbf{G}}^{\prime} v(\mathbf{G}) S(\mathbf{G}) .
$$

In the above equation, $\mathbf{G}$ corresponds to a plane-wave vector that is defined as $\mathbf{G}=\mathbf{g}+\Delta \mathbf{k}$, where $\mathbf{g}$ is a reciprocal lattice vector and $\Delta \mathbf{k}$ is the difference between any two Bloch wave vectors that are conventionally chosen to sample the first Brillouin zone. Note that $v(\mathbf{G})$ is the Coulomb kernel in reciprocal space that diverges at $\mathbf{G}=0$, making it numerically necessary to disregard this contribution to the sum, as indicated by the apostrophe. Thus, $S(\mathbf{G})$ is the partial functional derivative of the correlation energy with respect to $v(\mathbf{G})$, and we will return to its explicit definition later, as well as in Ref. [28] (see also Ref. [27]).

The thermodynamic limit is approached as $N \rightarrow \infty$, where $N$ is the number of particles in the simulation cell while the density is kept constant. Finite-size errors are defined as the difference between the thermodynamic limit and the finite simulation cell results. For electronic correlation energies obtained using many-electron perturbation theories, these errors typically decay as $1 / N$ as a consequence of long-range interatomic van der Waals forces. In the thermodynamic limit, $\sum_{\mathbf{G}}$ of Eq. (1) is replaced by $\int_{\mathbf{G}}$. Therefore, finite-size errors in the correlation energy of periodic systems originate from two sources [29]: (i) quadrature errors in the summation over $\mathbf{G}$, and (ii) the slow convergence of $S(\mathbf{G})$ with respect to the employed supercell size or $k$-point mesh. In the following, we discuss how to reduce both errors substantially.

We first seek to discuss finite-size errors originating from the quadrature in the summation over $\mathbf{G}$. These contributions can be partitioned into the $\mathbf{G}=0$ volume element contribution and the remaining terms. We note that, as a result of the Coulomb divergence, the integrable contribution of $S(\mathbf{0}) v(\mathbf{0})$ to the correlation energy is usually neglected in computer implementations of Eq. (1) $[11,27]$. However, this is the dominant contribution to the finite-size error of the correlation energy of insulators. A Taylor expansion of $S(\mathbf{G})$ around $\mathbf{G}=0$ shows that $S(\mathbf{G})$ exhibits a quadratic behavior close to zero, explaining the $1 / N$ decay of the finite-size error for three-dimensional insulators [27]. An estimate of $S(\mathbf{0}) v(\mathbf{0})$ can be obtained by spherically averaging $S(\mathbf{G})$ and interpolating around $\mathbf{G}=0$. Subsequently, the interpolated function is multiplied by the analytic Coulomb kernel and integrated over a sphere around $\mathbf{G}=0$, yielding an estimate of $S(\mathbf{0}) v(\mathbf{0})$ [27]. However, this approach is not well defined for anisotropic systems because it requires a spherical cutoff parameter. In this work, we propose to interpolate $S(\mathbf{G})$ using a tricubic interpolation without spherical averaging. Once obtained, the interpolation of $S(\mathbf{G})$ and the analytic expression for the Coulomb kernel allows for integrating over $\mathbf{G}$ on a very fine grid, simulating the thermodynamic limit integration. This approach accounts for the $S(\mathbf{0}) v(\mathbf{0})$ contribution to the correlation energy and reduces quadrature errors originating from too coarse a Brillouin zone sampling. We refer to coupled cluster correlation energies obtained using this interpolation strategy as CC-FS. 


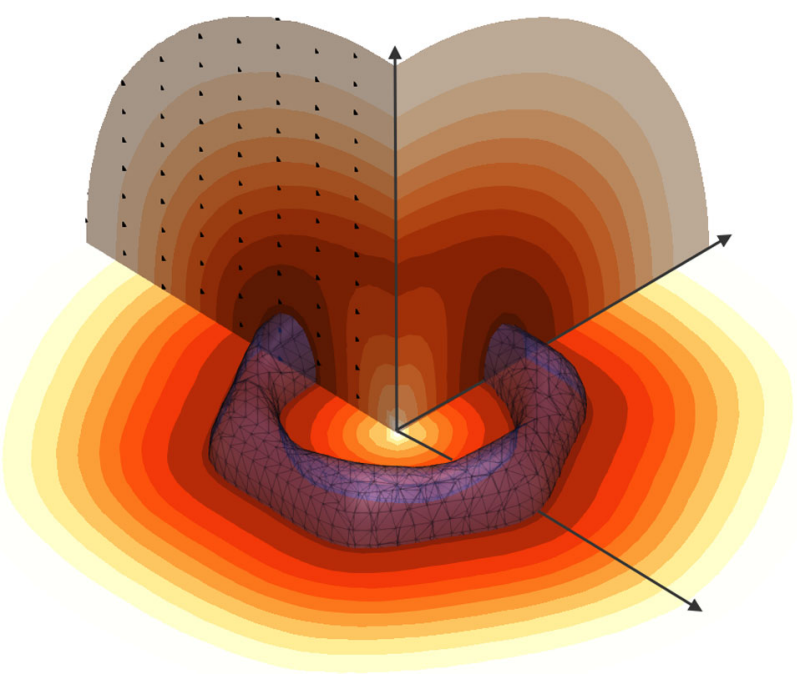

FIG. 1. Partial derivative of correlation energy with respect to the Coulomb kernel for graphite. Slices and isosurface of $S(\mathbf{G})$ for carbon graphite in the ABC stacking. Darker colors indicate more negative values. White corresponds to zero. The blue isosurface is a hexagonally shaped torus, and it reflects the anisotropic shape for $S(\mathbf{G})$. Black dots represent the sampling points using a $4 \times 4 \times 4 k$-point mesh.

To illustrate the importance of the interpolation method, we consider the following example. Figure 1 shows slices and an isosurface of the interpolated $S(\mathbf{G})$ for carbon graphite in the $\mathrm{ABC}$ stacking. Black dots indicate sampling points of $S(\mathbf{G})$ obtained using coupled cluster singles and doubles theory and a $4 \times 4 \times 4 k$-point mesh. This figure illustrates that $S(\mathbf{G})$ is very anisotropic around $\mathbf{G}=0$. Furthermore, we show that even a $4 \times 4 \times 4 k$-point mesh sampling, indicated by the black dots, corresponds to a relatively coarse grid, causing non-negligible quadrature errors. We return to the discussion of the results for the correlation energy later.

We now turn to the discussion of finite-size errors in semiconductors and metals. We stress that small gap systems suffer from a relatively slow convergence of $S(\mathbf{G})$ with respect to the studied system size. This behavior can be understood by considering the definition of $S(\mathbf{G})$ in second-order Møller-Plesset perturbation (MP2) theory,

$$
S(\mathbf{G})=\sum_{\mathbf{k}_{i}, \mathbf{k}_{j}, \mathbf{k}_{a}} \sum_{n_{i}, n_{j}, n_{a}, n_{b}} \frac{\Gamma_{i j}^{a b}(\mathbf{G})}{\epsilon_{i}+\epsilon_{j}-\epsilon_{a}-\epsilon_{b}},
$$

where $\epsilon_{i}$ correspond to one-electron energies usually obtained from Hartree-Fock theory. The indices $i, j$ and $a, b$ label occupied and virtual orbitals, respectively, and are understood to be a shorthand for the Bloch wave vector $\mathbf{k}_{i}$ and a band index $n_{i}$. Because of momentum conservation, $\mathbf{k}_{b}$ can be calculated from the other Bloch wave vectors in the above equation. Note that $\Gamma_{i j}^{a b}(\mathbf{G})$ is defined in Ref. [28]. The summation over Bloch vectors in Eq. (2) introduces quadrature errors that cause the slow convergence of $S(\mathbf{G})$ towards the thermodynamic limit. In the case of semiconductors or metals, these errors can become significant because $1 /\left(\epsilon_{i}+\epsilon_{j}-\epsilon_{a}-\epsilon_{b}\right)$ varies strongly depending on $\mathbf{k}_{i}, \mathbf{k}_{j}, \mathbf{k}_{a}$, and $\mathbf{k}_{b}$. In particular, materials with a Dirac cone at the Fermi surface such as graphene exhibit a large variation of the denominator between zero and several $\mathrm{eV}$ depending on $\mathbf{k}$. As a result, Eq. (2) needs to be calculated using a finer $k$-point mesh to reduce quadrature errors. In this work, we show that the above quadrature errors can be substantially reduced by calculating and averaging $S(\mathbf{G})$ for a set of shifted $k$-point meshes. Note that the vectors $\mathbf{G}$ are not affected by shifting the employed $k$-mesh because $\mathbf{G}$ depends only on the difference between any two Bloch wave vectors $\Delta \mathbf{k}$. We replace $S(\mathbf{G})$ in Eq. (1) with an average obtained for $N_{t}$ different $k$ meshes shifted from $\Gamma$ by $\mathbf{t}_{i}$ such that

$$
\bar{S}(\mathbf{G})=\frac{1}{N_{t}} \sum_{i=1}^{N_{t}} S_{\mathbf{t}_{i}}(\mathbf{G}) .
$$

The shifts $\mathbf{t}_{i}$ are chosen such that they sample the first Brillouin zone uniformly. Coupled cluster theory calculations for different shifts can be performed independently from each other, and the computational complexity scales only linearly with respect to $N_{t}$. Coupled cluster theory energies that have been obtained using this twist-averaging technique are referred to as CC-TA or CC-TA-FS if the interpolation method has been employed as well.

We note that quantum Monte Carlo (QMC) methods employ finite-size corrections that share similarities with the methods outlined above [29-31]. However, QMC methods such as diffusion Monte Carlo are real-space theories that provide estimates of total energies rather than partitioning the energy into a Hartree-Fock and an electronic correlation contribution. An advantage of the partitioning ansatz is that Hartree-Fock energy contributions can be converged to the thermodynamic limit independently from the correlation energy at little extra computational cost. Consequently, finite-size corrections are only required for the comparatively smaller correlation energy contributions. In passing, we note that auxiliary-field quantum Monte Carlo theory employs finite-size corrections that are based on parametrized density functionals obtained from finite, uniform, electron gas simulation cells [32]. Such corrections work for solids but have not yet been applied to surfaces or molecular crystals, where they are expected to be less accurate.

\section{RESULTS}

We now turn to the discussion of the results obtained using the methods outlined above. The present computations were performed using the VASP code $[33,34]$ and the projector augmented-wave method [35]. The coupled 


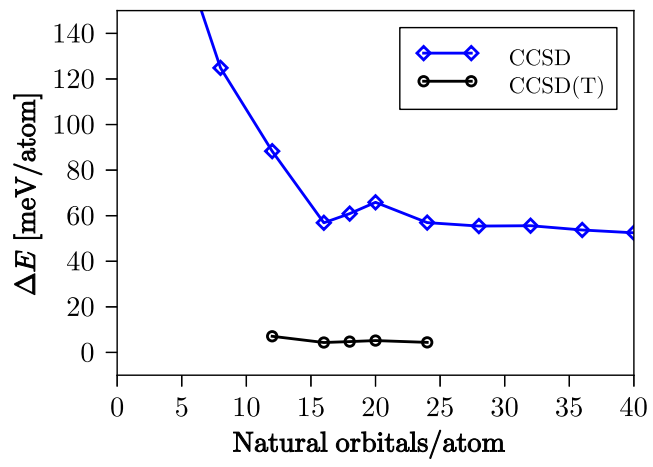

FIG. 2. Convergence of the energy difference between the carbon diamond and graphite using CCSD and 2 atomic unit cells with respect to the number of natural orbitals used. The (T) correlation energy contribution has been added to the CCSD energy using 16 natural orbitals per atom.

cluster theory calculations were partly performed using the newly developed cc4s code [36] interfaced with VASP and employing the automated tensor contraction engine CTF [37]. More technical details are outlined in Ref. [28].

As a first application, we investigate the carbon diamond and graphite crystals. Before discussing the thermodynamic limit convergence, we seek to address the convergence of the calculated correlation energy differences with respect to the employed orbital basis. We employ MP2 natural orbitals that are obtained using a procedure outlined in Ref. [38]. Figure 2 shows the convergence of coupled cluster singles and doubles (CCSD) and perturbative triples (T) correlation energy differences with respect to the number of bands using a $2 \times 2 \times 2 k$-point mesh, respectively. We find that calculations using 16 orbitals per carbon atom yield an energy difference that agrees to within $4 \mathrm{meV} /$ atom compared to results obtained using 40 natural orbitals per atom. The (T) correction to CCSD converges even faster with respect to the number of orbitals and is fortuitously close to zero in the case of the $2 \times 2 \times 2 k$ point mesh. We stress that natural orbitals allow for a significantly more systematic truncatability and improved basis-set incompleteness error cancellation between different systems compared to virtual Hartree-Fock or density functional theory orbitals. Achieving the same level of accuracy requires several hundred virtual Hartree-Fock orbitals per atom. Convergence with respect to other computational parameters has been checked and is discussed in Ref. [28].

We now turn to the discussion of finite-size errors in total correlation energies. The top and middle panels in Fig. 3 show CCSD correlation energies retrieved as a function of the number of $k$ points of graphite and diamond, respectively. We note that twist averaging (TA) is necessary for CCSD correlation energies to achieve a smooth $1 / N$ convergence to the thermodynamic limit, in particular, for graphite. Accounting for quadrature errors by means of the tricubic interpolation method (CCSD-TA-FS) yields

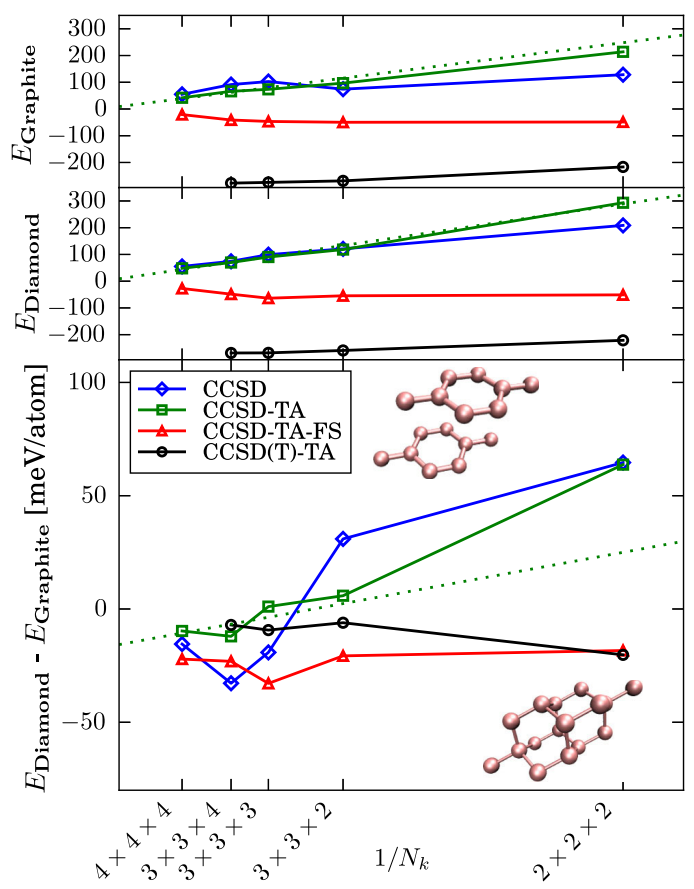

FIG. 3. Energy difference between carbon diamond and graphite phases. The dotted line represents the linear fit of the uncorrected values with shifts. (T) corrections (black) are on top of CCSD-TA-FS energy obtained using a $4 \times 4 \times 4 k$-point mesh (red). Zero-point energies are included, and they stabilize graphite compared to diamond by $9 \mathrm{meV} /$ atom.

rapidly convergent correlation energies for both carbon diamond and graphite. CCSD-TA-FS correlation energies obtained using a $2 \times 2 \times 2 k$ mesh only deviate from extrapolated CCSD thermodynamic limit energies by approximately $60 \mathrm{meV} /$ atom. We note that correlation energies obtained using the same $k$ mesh and CCSD-TA theory exhibit finite-size errors on the scale of 200-300 meV/atom. The CCSD(T) correlation energies are obtained using twist averaging for the (T) contribution and adding the correction to the CCSD-TA-FS result obtained using a $4 \times 4 \times 4 k$-point mesh. This allows for investigating the finite-size errors of the $(\mathrm{T})$ correction independently from finite-size errors of CCSD theory. We find that $(\mathrm{T})$ converges rapidly with respect to the employed $k$-mesh size, reflecting its short-ranged nature.

The bottom panel in Fig. 3 shows the differences of the total energies of both carbon allotropes, including zeropoint corrections retrieved as a function of the employed $k$ point mesh. Results obtained using CCSD theory without finite-size corrections are depicted by the blue line and oscillate strongly with increasing $k$-point mesh density. By using too-coarse $k$-point meshes, we can predict graphite to be more stable than diamond, whereas denser $k$-point meshes predict diamond to be the more stable allotrope. Employing the averaging over different shifts yields CCSD-TA results that converge significantly smoother with increasing $k$-point mesh density as shown by the 
green line. Furthermore, performing the newly proposed tricubic interpolation and integration in addition to the twist averaging referred to as CCSD-TA-FS yields rapidly convergent energy differences shown by the red line. We note that CCSD-TA-FS using a $2 \times 2 \times 2 k$-point mesh is as close to the thermodynamic limit as CCSD-TA using a $4 \times 4 \times 4 k$-point mesh. Since the computational complexity scales at least as $\mathcal{O}\left(N_{k}^{4}\right)$ with respect to the number of $k$ points, this corresponds to a reduction in the computational cost by 3 orders of magnitude. From these calculations, we conclude that CCSD theory predicts diamond to be more stable than graphite by $22 \mathrm{meV} /$ atom, including zero-point energies. We have also performed perturbative triples calculations and added the corresponding correlation energy correction to our CCSD findings. CCSD(T) theory predicts graphite to be slightly less stable than diamond by $7 \mathrm{meV} /$ atom, including zero-point corrections. We note that our $\operatorname{CCSD}(\mathrm{T})$ results agree with experimental findings to within the observed precision of $\operatorname{CCSD}(\mathrm{T})$ for similar applications, which is generally better than $1 \mathrm{kcal} / \mathrm{mol}$ (43 meV/atom). The difference in the experimental Gibbs free energy of carbon diamond and graphite at room temperature has been reported to be $25 \mathrm{meV} /$ atom [39], predicting graphite to be more stable than diamond.

Having demonstrated the ability of the proposed method to correct for finite-size errors on the scale of about $100 \mathrm{meV} /$ atom, we now study the thermodynamic limit of the cohesive energy of the weakly bound neon solid. In this case, we need to correct for finite-size errors on the scale of a few meV/atom. The dominant contribution to the attractive long-range interatomic interaction of neon atoms originates from van der Waals forces. Table I summarizes MP2, CCSD, and CCSD(T) cohesive energies obtained with and without the proposed finite-size correction. The correction yields MP2 and CCSD cohesive energies using $3 \times 3 \times 3 k$ meshes that deviate from the thermodynamic limit results by approximately $1-2 \mathrm{meV} /$ atom, whereas the uncorrected estimates deviate by $2-4 \mathrm{meV} /$ atom. Although the finite-size errors are small on an absolute scale, we stress that the corresponding relative finite-size errors of the cohesive energy are non-negligible. Our best estimates for the MP2, CCSD, and CCSD(T) cohesive energies using finite-size corrections and a $4 \times 4 \times 4 k$ mesh agree with

TABLE I. Cohesive energies of solid neon obtained using MP2, CCSD, and CCSD(T) theory. The summarized results have been extrapolated to the complete basis set limit using pseudized augcc-pV(D,T)Z basis sets and corrected for basis-set superposition errors using counterpoise corrections. All units are in meV/atom.

\begin{tabular}{lccccc}
\hline \hline$k$ mesh & MP2 & MP2-FS & CCSD & CCSD-FS & CCSD(T)-FS \\
\hline $2 \times 2 \times 2$ & -5 & 25 & 4 & 36 & 47 \\
$3 \times 3 \times 3$ & 13 & 17 & 16 & 21 & 32 \\
$4 \times 4 \times 4$ & 17 & 17 & 19 & 19 & 30 \\
Ref. $[10]$ & & 19 & & 22 & 27 \\
\hline \hline
\end{tabular}

results obtained using the incremental method to within $3 \mathrm{meV} /$ atom [6,10]. Furthermore, $\operatorname{CCSD}(\mathrm{T})$ predicts a cohesive energy of $30 \mathrm{meV} /$ atom, which is in good agreement with experimental estimates of $27 \mathrm{meV} /$ atom corrected for zero-point fluctuations [6].

As a final demonstration of the applicability of the proposed method to reach the thermodynamic limit, we study the adsorption energy of a single water molecule on an $h$-BN sheet. The same system has recently been studied using diffusion Monte Carlo (DMC), the random-phase approximation (RPA), and dispersion functionals [40,41], as well as molecular MP2 [42] and periodic coupled cluster theory [43], demonstrating the need for reliable methods that can account for long-range van der Waals interactions and also to provide benchmark data. Furthermore, the recent work of Al-Hamdani et al. [40] illustrates the importance of long-range correlation effects that account for approximately $25 \%$ of the reference adsorption energy computed in a $(4 \times 4)$ unit cell of $h$-BN. Figure 4 shows calculated adsorption energies at the level of RPA plus second-order screened exchange, MP2, CCSD, and $\operatorname{CCSD}(\mathrm{T})$ theories retrieved as a function of the number of atoms in the $h$-BN sheet. Convergence with respect to other computational parameters has been checked and is discussed in Ref. [28]. Using MP2 theory, it is possible to study very large systems [40], and we find that the MP2 adsorption energy converges slowly to a thermodynamic limit value of $119 \mathrm{meV}$. We note that finite-size errors for adsorption energies on two-dimensional insulators are expected to decay as $1 / N^{2}$, which is the predicted scaling from pairwise additive van der Waals interactions [40]. Applying the proposed finite-size correction to MP2 theory for the $(4 \times 4)$ unit cell $h$-BN sheet with 32 atoms yields an

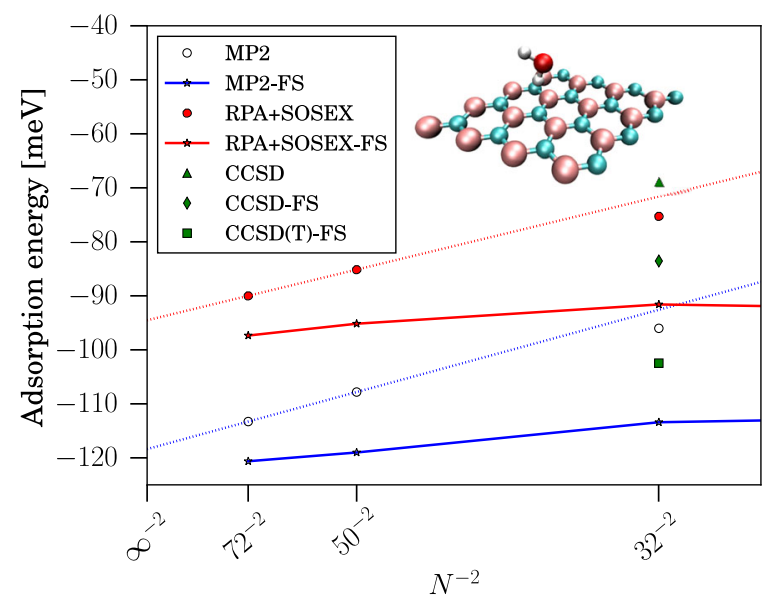

FIG. 4. Adsorption energy of a single water molecule on an $h$-BN sheet. The energies are retrieved as a function of atoms in the sheet using MP2, RPA + SOSEX, CCSD, and CCSD(T) theory. FS indicates that finite-size corrections are included. The results have been obtained using pseudized aug-cc-pVTZ basis sets and corrected for basis-set superposition errors using counterpoise corrections. 
adsorption energy of $113 \mathrm{meV}$, in close agreement with the thermodynamic limit result. We observe a similar speed-up in convergence using RPA + SOSEX-FS theory, illustrating the transferability of the proposed method. The water adsorption on the 32-atom $h$-BN sheet can also be studied using the more sophisticated CCSD theory [43]. CCSD with and without finite-size corrections yields an adsorption energy of $83 \mathrm{meV}$ and $68 \mathrm{meV}$, respectively. The finitesize corrections of MP2 and CCSD theory agree to within a few meV. However, we note that CCSD theory underbinds the water molecule. We estimate the $(\mathrm{T})$ contribution using the 18-atom cell only and find that $\operatorname{CCSD}(\mathrm{T})$ theory yields adsorption energy of $102 \mathrm{meV}$ and $87 \mathrm{meV}$ with and without finite-size corrections, respectively. The DMC adsorption energy was reported to be $84 \mathrm{meV}$ without finite-size corrections and agrees well with our $\operatorname{CCSD}(\mathrm{T})$ results using the same 32-atom $h$-BN sheet, disregarding finite-size corrections.

\section{CONCLUSION AND OUTLOOK}

In conclusion, we have introduced an efficient and accurate thermodynamic limit correction for wavefunction-based theory calculations of solids and surfaces that is free of adjustable parameters and easy to implement. We have demonstrated that this correction allows for reducing the computational cost by several orders of magnitude without compromising accuracy. We have studied ground-state problems, where the convergence to the thermodynamic limit is crucial and finite-size errors span a range of 1-100 meV/atom. Despite the local character of electronic correlation, we stress that a proper treatment of long-range correlation effects is of paramount importance for reliable and highly accurate many-electron theories in condensed-matter systems. We have applied the proposed finite-size correction in combination with the gold standard of quantum chemistry $\operatorname{CCSD}(\mathrm{T})$ theory to calculate the cohesive energy of the Ne solid, and the energy difference between carbon diamond and graphite crystals, as well as the adsorption of a water molecule on an $h$-BN sheet. In general, our $\operatorname{CCSD}(\mathrm{T})$ results are in good agreement with experimental findings and DMC results. This paves the way for a routine use of highly accurate coupled cluster theories in the field of surface science and solid-state physics. We believe that the ability to predict accurate benchmark results will help the entire electronic structure theory community to improve further upon computationally efficient $a b$ initio theories and to help interpret experimental findings more reliably. To expand the scope of the proposed techniques even further, we will aim at combining them with explicit correlation and low-rank factorization methods [43-45].

In future studies, we will extend the proposed finite-size corrections to the study of excited states and metallic systems. We note that excited states and spectral functions can be calculated in the framework of equation-of-motion coupled cluster theory for solids, yielding excited-state structure factors that are expected to exhibit similar finitesize errors [46]. In metallic systems, the structure factor is still algebraic around $\mathbf{G}=0$. We are therefore confident that the proposed methods can also be transferred to the study of such systems, and we expect that the outlined twist-averaging methodology will be of significant importance when approaching the thermodynamic limit. We note, however, that the perturbative triples $(\mathrm{T})$ contribution requires methodological improvements when applied to metals.

\section{ACKNOWLEDGMENTS}

This project has received funding from the European Research Council (ERC) under the European Unions Horizon 2020 research and innovation program (Grant Agreement No 715594). The computational results presented have been achieved in part using the Vienna Scientific Cluster (VSC).

[1] J. Sun, R. C. Remsing, Y. Zhang, Z. Sun, A. Ruzsinszky, H. Peng, Z. Yang, A. Paul, U. Waghmare, X. Wu, M. L. Klein, and J. P. Perdew, Accurate First-Principles Structures and Energies of Diversely Bonded Systems from an Efficient Density Functional, Nat. Chem. 8, 831 (2016).

[2] W. M. C. Foulkes, L. Mitas, R. J. Needs, and G. Rajagopal, Quantum Monte Carlo Simulations of Solids, Rev. Mod. Phys. 73, 33 (2001).

[3] J. Yang, W. Hu, D. Usvyat, D. Matthews, M. Schütz, and G. K.-L. Chan, Ab Initio Determination of the Crystalline Benzene Lattice Energy to Sub-kilojoule/Mole Accuracy, Science 345, 640 (2014).

[4] G. H. Booth, A. Grüneis, G. Kresse, and A. Alavi, Towards an Exact Description of Electronic Wavefunctions in Real Solids, Nature (London) 493, 365 (2013).

[5] S. J. Nolan, M. J. Gillan, D. Alfè, N. L. Allan, and F. R. Manby, Calculation of Properties of Crystalline Lithium Hydride Using Correlated Wave Function Theory, Phys. Rev. B 80, 165109 (2009).

[6] K. Rościszewski, B. Paulus, P. Fulde, and H. Stoll, Ab Initio Calculation of Ground-State Properties of Rare-Gas Crystals, Phys. Rev. B 60, 7905 (1999).

[7] J. J. Shepherd, T. M. Henderson, and G. E. Scuseria, RangeSeparated Brueckner Coupled Cluster Doubles Theory, Phys. Rev. Lett. 112, 133002 (2014).

[8] R. Martinez-Casado, D. Usvyat, L. Maschio, G. Mallia, S. Casassa, J. Ellis, M. Schütz, and N. M. Harrison, Approaching an Exact Treatment of Electronic Correlations at Solid Surfaces: The Binding Energy of the Lowest Bound State of Helium Adsorbed on MgO(100), Phys. Rev. B 89, 205138 (2014).

[9] A. Hermann and P. Schwerdtfeger, Ground-State Properties of Crystalline Ice from Periodic Hartree-Fock Calculations and a Coupled-Cluster-Based Many-Body Decomposition of the Correlation Energy, Phys. Rev. Lett. 101, 183005 (2008). 
[10] P. Schwerdtfeger, B. Assadollahzadeh, and A. Hermann, Convergence of the M $\phi$ ller-Plesset Perturbation Series for the fcc Lattices of Neon and Argon, Phys. Rev. B 82, 205111 (2010).

[11] J. McClain, Q. Sun, Garnet K.-L. Chan, and T. C. Berkelbach, Gaussian-Based Coupled-Cluster Theory for the Ground State and Band Structure of Solids, J. Chem. Theory Comput. 13, 1209 (2017).

[12] M. Motta, D. M. Ceperley, G. K.-L. Chan, J. A. Gomez, E. Gull, S. Guo, C. A. Jiménez-Hoyos, T. N. Lan, J. Li, F. Ma, A. J. Millis, N. V. Prokof'ev, U. Ray, G. E. Scuseria, S. Sorella, E. M. Stoudenmire, Q. Sun, I. S. Tupitsyn, S. R. White, D. Zgid, and S. Zhang (Simons Collaboration on the Many-Electron Problem), Towards the Solution of the Many-Electron Problem in Real Materials: Equation of State of the Hydrogen Chain with State-of-the-Art ManyBody Methods, Phys. Rev. X 7, 031059 (2017).

[13] J. P. F. LeBlanc, A. E. Antipov, F. Becca, I. W. Bulik, G. K.-L. Chan, C.-M. Chung, Y. Deng, M. Ferrero, T. M. Henderson, C. A. Jiménez-Hoyos, E. Kozik, X.-W. Liu, A. J. Millis, N. V. Prokof'ev, M. Qin, G. E. Scuseria, H. Shi, B. V. Svistunov, L. F. Tocchio, I. S. Tupitsyn, S. R. White, S. Zhang, B.-X. Zheng, Z. Zhu, and E. Gull (Simons Collaboration on the Many-Electron Problem), Solutions of the Two-Dimensional Hubbard Model: Benchmarks and Results from a Wide Range of Numerical Algorithms, Phys. Rev. X 5, 041041 (2015).

[14] A. Grüneis, J. J. Shepherd, A. Alavi, D. P. Tew, and G. H. Booth, Explicitly Correlated Plane Waves: Accelerating Convergence in Periodic Wavefunction Expansions, J. Chem. Phys. 139, 084112 (2013).

[15] C. Hättig, D. P. Tew, and B. Helmich, Local Explicitly Correlated Second- and Third-Order M $\phi$ llerplesset Perturbation Theory with Pair Natural Orbitals, J. Chem. Phys. 136, 204105 (2012).

[16] C. Riplinger and F. Neese, An Efficient and Near Linear Scaling Pair Natural Orbital Based Local Coupled Cluster Method, J. Chem. Phys. 138, 034106 (2013).

[17] H.-J. Werner and M. Schütz, An Efficient Local Coupled Cluster Method for Accurate Thermochemistry of Large Systems, J. Chem. Phys. 135, 144116 (2011).

[18] D. Usvyat, L. Maschio, and M. Schütz, Periodic Local MP2 Method Employing Orbital Specific Virtuals, J. Chem. Phys. 143, 102805 (2015).

[19] G. Knizia and G. K.-L. Chan, Density Matrix Embedding: A Simple Alternative to Dynamical Mean-Field Theory, Phys. Rev. Lett. 109, 186404 (2012).

[20] I. W. Bulik, G. E. Scuseria, and J. Dukelsky, Density Matrix Embedding from Broken Symmetry Lattice Mean Fields, Phys. Rev. B 89, 035140 (2014).

[21] F. R. Manby, M. Stella, J. D. Goodpaster, and T. F. Miller, A Simple, Exact Density-Functional-Theory Embedding Scheme, J. Chem. Theory Comput. 8, 2564 (2012).

[22] A. A. Rusakov, J. J. Phillips, and D. Zgid, Local Hamiltonians for Quantitative Green's Function Embedding Methods, J. Chem. Phys. 141, 194105 (2014).

[23] N. Govind, Y. A. Wang, and E. A. Carter, ElectronicStructure Calculations by First-Principles Density-Based Embedding of Explicitly Correlated Systems, J. Chem. Phys. 110, 7677 (1999).
[24] F. R. Manby, M. Stella, J. D. Goodpaster, and T. F. Miller, A Simple, Exact Density-Functional-Theory Embedding Scheme, J. Chem. Theory Comput. 8, 2564 (2012).

[25] J. F. Dobson, A. White, and A. Rubio, Asymptotics of the Dispersion Interaction: Analytic Benchmarks for van der Waals Energy Functionals, Phys. Rev. Lett. 96, 073201 (2006).

[26] S. Chiesa, D. M. Ceperley, R. M. Martin, and M. Holzmann, Finite-Size Error in Many-Body Simulations with LongRange Interactions, Phys. Rev. Lett. 97, 076404 (2006).

[27] K. Liao and A. Grüneis, Communication: Finite Size Correction in Periodic Coupled Cluster Theory Calculations of Solids, J. Chem. Phys. 145, 141102 (2016).

[28] See Supplemental Material at http://link.aps.org/ supplemental/10.1103/PhysRevX.8.021043 for additional computational details.

[29] M. Holzmann, R. C. Clay, M. A. Morales, N. M. Tubman, D. M. Ceperley, and C. Pierleoni, Theory of Finite Size Effects for Electronic Quantum Monte Carlo Calculations of Liquids and Solids, Phys. Rev. B 94, 035126 (2016).

[30] C. Lin, F. H. Zong, and D. M. Ceperley, Twist-Averaged Boundary Conditions in Continuum Quantum Monte Carlo Algorithms, Phys. Rev. E 64, 016702 (2001).

[31] C. Filippi and D. Ceperley, Quantum Monte Carlo Calculation of Compton Profiles of Solid Lithium, Phys. Rev. B 59, 7907 (1999).

[32] H. Kwee, S. Zhang, and H. Krakauer, Finite-Size Correction in Many-Body Electronic Structure Calculations, Phys. Rev. Lett. 100, 126404 (2008).

[33] G. Kresse and J. Hafner, Norm-Conserving and Ultrasoft Pseudopotentials for First-Row and Transition Elements, J. Phys. Condens. Matter 6, 8245 (1994).

[34] G. Kresse and J. Furthmüller, Efficient Iterative Schemes for Ab Initio Total-Energy Calculations Using a Plane-Wave Basis Set, Phys. Rev. B 54, 11169 (1996).

[35] P. E. Blöchl, Projector Augmented-Wave Method, Phys. Rev. B 50, 17953 (1994).

[36] The details about the cc4s code will be elaborated on in a future paper.

[37] E. Solomonik, D. Matthews, J. R. Hammond, J. F. Stanton, and J. Demmel, A Massively Parallel Tensor Contraction Framework for Coupled-Cluster Computations, J. Parallel Distrib. Comput. 74, 3176 (2014).

[38] A. Grüneis, G. H. Booth, M. Marsman, J. Spencer, A. Alavi, and G. Kresse, Natural Orbitals for Wave Function Based Correlated Calculations Using a Plane Wave Basis Set, J. Chem. Theory Comput. 7, 2780 (2011).

[39] D. D. Wagman, J. E. Kilpatrick, W. J. Taylor, K. S. Pitzer, and F. D. Rossini, Heats, Free Energies, and Equilibrium Constants of Some Reactions Involving $\mathrm{O}_{2}, \mathrm{H}_{2}, \mathrm{H}_{2} \mathrm{O}, C, C o$, $\mathrm{Co}_{2}$, and $\mathrm{Ch}_{4}$, J. Res. Natl. Bur. Stand. 34, 143 (1945).

[40] Y. S. Al-Hamdani, M. Rossi, D. Alfè, T. Tsatsoulis, B. Ramberger, J. G. Brandenburg, A. Zen, G. Kresse, A. Grüneis, A. Tkatchenko, and A. Michaelides, Properties of the Water to Boron Nitride Interaction: From Zero to Two Dimensions with Benchmark Accuracy, J. Chem. Phys. 147, 044710 (2017).

[41] Y. Wu, L. K. Wagner, and N. R. Aluru, Hexagonal Boron Nitride and Water Interaction Parameters, J. Chem. Phys. 144, 164118 (2016). 
[42] Y. Wu, L. K. Wagner, and N. R. Aluru, The Interaction between Hexagonal Boron Nitride and Water from First Principles, J. Chem. Phys. 142, 234702 (2015).

[43] F. Hummel, T. Tsatsoulis, and A. Grüneis, Low Rank Factorization of the Coulomb Integrals for Periodic Coupled Cluster Theory, J. Chem. Phys. 146, 124105 (2017).

[44] A. Grüneis, Efficient Explicitly Correlated Many-Electron Perturbation Theory for Solids: Application to the Schottky Defect in MgO, Phys. Rev. Lett. 115, 066402 (2015).
[45] A. Grüneis, S. Hirata, Y.y. Ohnishi, and S. Ten-no, Perspective: Explicitly Correlated Electronic Structure Theory for Complex Systems, J. Chem. Phys. 146, 080901 (2017).

[46] J. McClain, J. Lischner, T. Watson, D. A. Matthews, E. Ronca, S. G. Louie, T. C. Berkelbach, and G. K.-L. Chan, Spectral Functions of the Uniform Electron Gas via Coupled-Cluster Theory and Comparison to the GW and Related Approximations, Phys. Rev. B 93, 235139 (2016). 